\title{
PHOTOGRAPHIC GUIDE TO MEDIAN STUBBLE HEIGHTS
}

\section{BUREAU OF LAND MANAGEMENT}

Challis Resource Area

Salmon, Idaho 


\section{BLM LIBRARY \\ BLDG 50, ST-150A \\ DENVER FEDCRAL CENTER \\ P. O. BOX 25047 \\ DENVER, COLORADO 80225}

\section{Acknowledgments}

This photographic field guide was prepared by the staff of the Bureau of Land Management's, Challis Resource Area to provide a practical monitoring tool for determining stubble height on riparian areas. In particular, the staff recognizes David Blackstun for analyzing the field data and developing the procedure for using the median instead of the average stubble height and Dianne Simodynes for compiling the photographs for the publication. Thanks to Melinda Ritacco and Ervin Cowley for formatting and preparation for publication.






\section{8}

\section{Contents}

Page

Riparian Monitoring and Median Stubble Height $\ldots \ldots \ldots \ldots \ldots \ldots$

Figure 1: Form for data collection and determining median stubble height $\ldots . .2$

Median Stubble Height -1 inch $\ldots \ldots \ldots \ldots \ldots \ldots \ldots \ldots \ldots \ldots$

Median Stubble Height -2 inches $\ldots \ldots \ldots \ldots \ldots \ldots \ldots \ldots \ldots$

Median Stubble Height -3 inches $\ldots \ldots \ldots \ldots \ldots \ldots \ldots \ldots \ldots$

Median Stubble Height -4 inches, Sedge . . . . . . . . . . . . . 9

Median Stubble Height -4 inches, Grass/Forb . . . . . . . . . . . . 11

Median Stubble Height -5 inches, Sedge . . . . . . . . . . . 13

Median Stubble Height -5 inches, Grass/Forb . . . . . . . . . . . 15

Median Stubble Height -6 inches $\ldots \ldots \ldots \ldots \ldots \ldots \ldots \ldots \ldots$

Median Stubble Height -7 inches $\ldots \ldots \ldots \ldots \ldots \ldots \ldots \ldots$

Median Stubble Height -8 inches $\ldots \ldots \ldots \ldots \ldots \ldots \ldots \ldots \ldots \ldots$

Location Index of Key Area Sites . . . . . . . . . . . . . . 23 


\section{Riparian Monitoring and Median Stubble Height}

Key areas have been established to monitor effects of grazing on riparian and aquatic habitats. The locations for potential key sites were inspected by interdisciplinary teams comprised of BLM staff representing various expertise to determine if the a site was representative of the entire habitat. Key areas are advantageous because they provide a good indication of grazing use without having to monitor the entire area being grazed.

Stubble height measurements are a simple and effective tool to monitor rangeland use in these key areas. Individual plant measurements are collected from herbaceous vegetation such as grasses, sedges, and rushes, while excluding forbs. Generally stubble heights of 4 to 6 inches are an acceptable standard. Plants with this much height remaining usually provide effective streambank protection, prevent sedimentation, and maintain or improve plant communities.

Within the key area, measurements are taken on both sides of the stream, along the greenline, usually within 3 feet of the waterline. Fifty measurements (using a pace point method) on each side of the stream for a total of 100 samples. At each pace, the plant or cluster of plants located at the tip of the observers toe is measured to the nearest one-half inch.

Once all samples have been collected, the median stubble height value is determined. The samples are ranked in descending order from the smallest to the highest value. The median value is the single mid-point for and odd number of samples and the average of the two mid-point values. For example, in a even numbered data set, 100 samples, the $50^{\text {th }}$ and $51^{\text {st }}$ values would be averaged giving the median value (see Figure 1).

A median value provides a better representation of the overall community than the average. Usually there is not a normal distribution of data points. That is the number of short stubble heights or tall stubble height are not evenly distributed. In some cases, extra tall plants may over influence the average number.

For instance, fifteen stubble height measurements provided the following data: .5", 1", 1", 4", 4", 4", 5", 6", 6", 6", 7", 7", 7", 7", 7". The average for the sample would be 4.8 inches. However, the median is 6.0 inches. The average is more sensitive to extremes making the median more accurate without complicated statistical analysis.

\section{References:}

a. Challis Resource Area Monitoring Procedures, April 1996

b. Statistical Evaluation of Stubble Height Data, David E. Blackstun 
Figure 1: Form used for data collection and determining median stubble heights.

\begin{tabular}{|c|c|c|c|c|c|c|c|c|c|c|}
\hline \multicolumn{11}{|l|}{ Allotment: } \\
\hline \multicolumn{11}{|l|}{ Pasture: } \\
\hline \multicolumn{11}{|l|}{ Key Area: } \\
\hline \multicolumn{11}{|l|}{ Observers: } \\
\hline & \multicolumn{2}{|c|}{ Stubble } & \multicolumn{2}{|c|}{ Stubble } & \multicolumn{2}{|c|}{ Stubble } & \multicolumn{2}{|c|}{ Stubble } & \multicolumn{2}{|c|}{ Stubble } \\
\hline & \multicolumn{2}{|c|}{ Measurement } & \multicolumn{2}{|c|}{ Measurement } & \multicolumn{2}{|c|}{ Measurement } & \multicolumn{2}{|c|}{ Measurement } & \multicolumn{2}{|c|}{ Measurement } \\
\hline & \multicolumn{2}{|c|}{ Date } & \multicolumn{2}{|c|}{ Date } & \multicolumn{2}{|c|}{ Date } & \multicolumn{2}{|c|}{ Date } & \multicolumn{2}{|c|}{ Example } \\
\hline & & & & & & & & & & \\
\hline Inches & $P B$ & LB & $\mathrm{RB}$ & LB & $P B$ & LB & $R B$ & LB & $\mathrm{PB}$ & LB \\
\hline \multicolumn{11}{|l|}{0.5} \\
\hline \multicolumn{11}{|l|}{1.0} \\
\hline 1.5 & & & & & & & & & 11 & \\
\hline 2.0 & & & & & & & & & III & \\
\hline 2.5 & & & & & & & & & III & \\
\hline 3.0 & & & & & & & & & III & 1 \\
\hline 3.5 & & & & & & & & & $\ln \times 1$ & II \\
\hline 4.0 & & & & & & & & & Imxy & III \\
\hline 4.5 & & & & & & & & & |rk| & 稌1 \\
\hline 5.0 & & & & & & & & & IxN1 & III \\
\hline 5.5 & & & & & & & & & IIII & IxI \\
\hline 6.0 & & & & & & & & & IIII & XIIII \\
\hline 6.5 & & & & & & & & & 1 & 妙1 \\
\hline 7.0 & & & & & & & & & 1 & IIII \\
\hline 7.5 & & & & & & & & & & IIII \\
\hline 8.0 & & & & & & & & & & III \\
\hline 8.5 & & & & & & & & & 11 & II \\
\hline 9.0 & & & & & & & & & 1 & in \\
\hline 9.5 & & & & & & & & & & 1 \\
\hline \multicolumn{11}{|l|}{10.0} \\
\hline 10.5 & & & & & & & & & & II \\
\hline 11.0 & & & & & & & & & & \\
\hline 11.5 & & & & & & & & & & \\
\hline 12.0 & & & & & & & & & & 11 \\
\hline$>12$ & & & & & & & & & 1 & 1 \\
\hline Totall & & & & & & & & & 50 & 50 \\
\hline Bank Median & & & & & & & &  & 4.0 & 6.0 \\
\hline Total Medianl & & & & & & & & 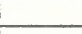 & 5.0 & $i$ \\
\hline Notesl & & & & & & & & & 1 & \\
\hline & & & & & & & & & & \\
\hline & & & & & & & & & & \\
\hline & & & & & & & & & & \\
\hline & & & & & & & & & & \\
\hline & & & & & & & & & & \\
\hline & & & & & & & & & & \\
\hline
\end{tabular}




\section{Median Stubble Height - 1 inch}

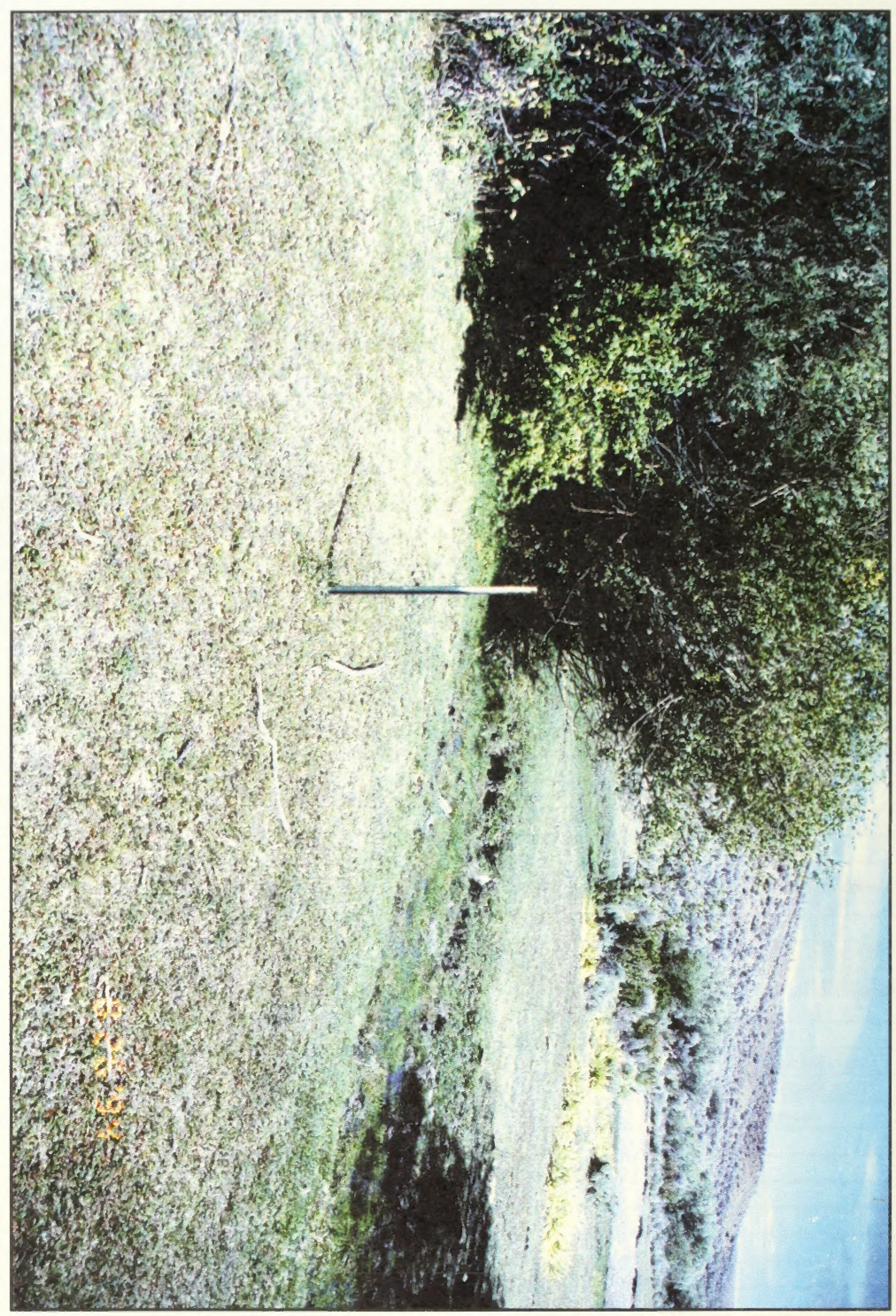

CBC-KA1, Upper Marker, Near View, 6/27/96 


\section{Median Stubble Height - 1 inch}

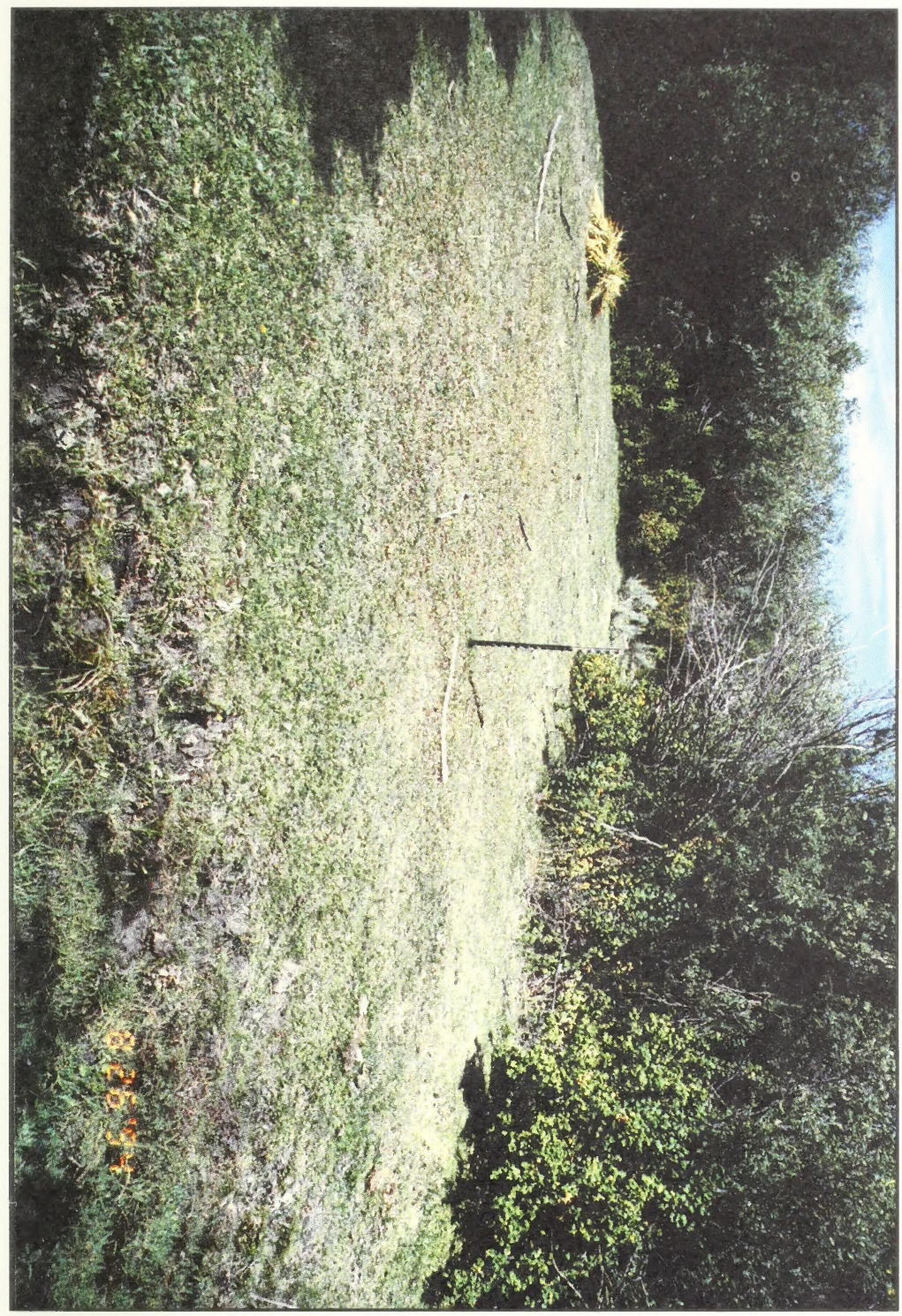

CBC-KA1, Upper Marker, Wide View, 8/26/94 


\section{Median Stubble Height - 2 inches}



LHC-KA3, Lower Marker, Near View, 9/26/95 




LHC-KA3, Lower Marker, Wide View, 9/26/95 


\section{Median Stubble Height - 3 inches}

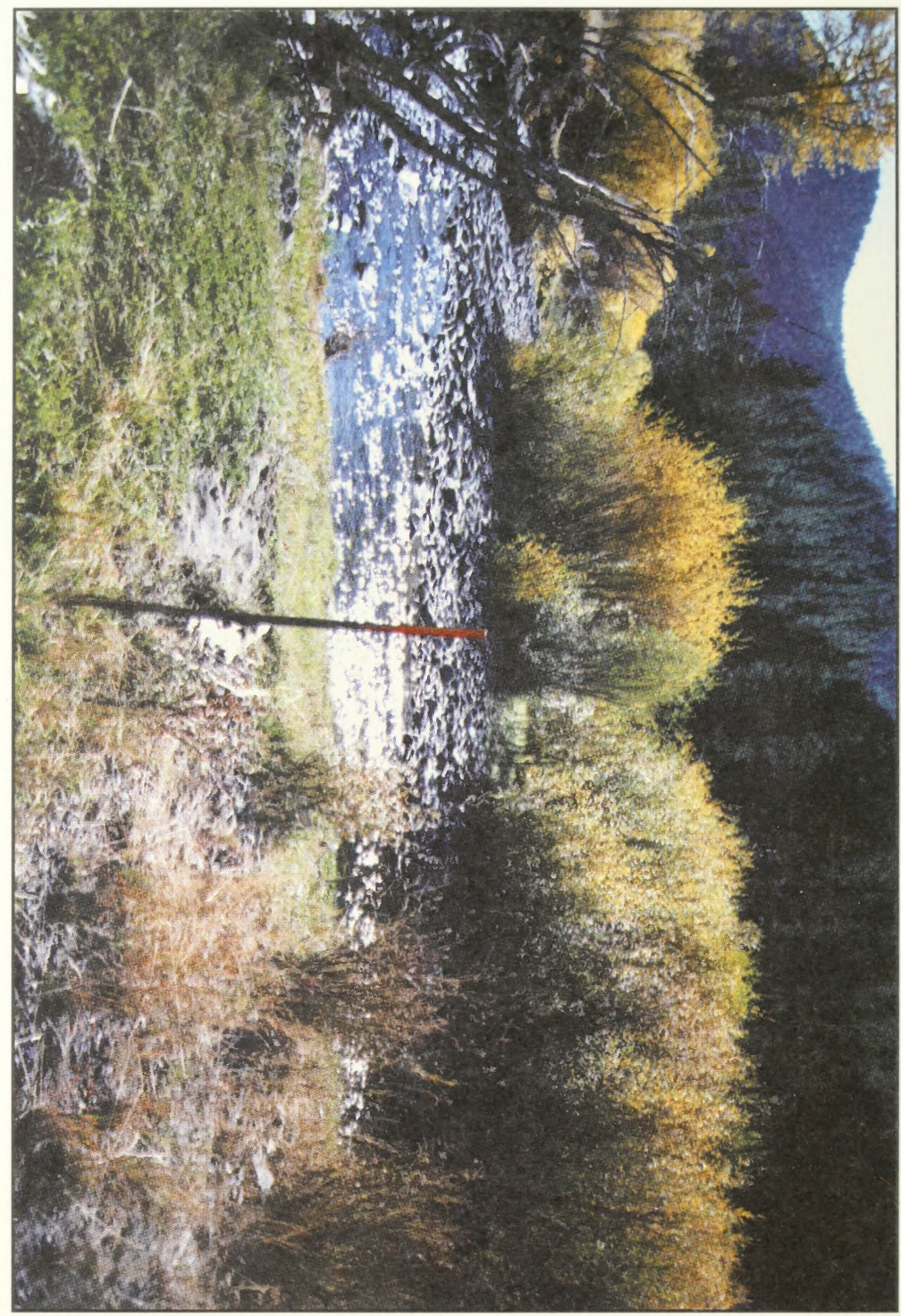

TC-KA2, Photo Point 1, Near View, 10/3/96 


\section{Median Stubble Height - 3 inches}

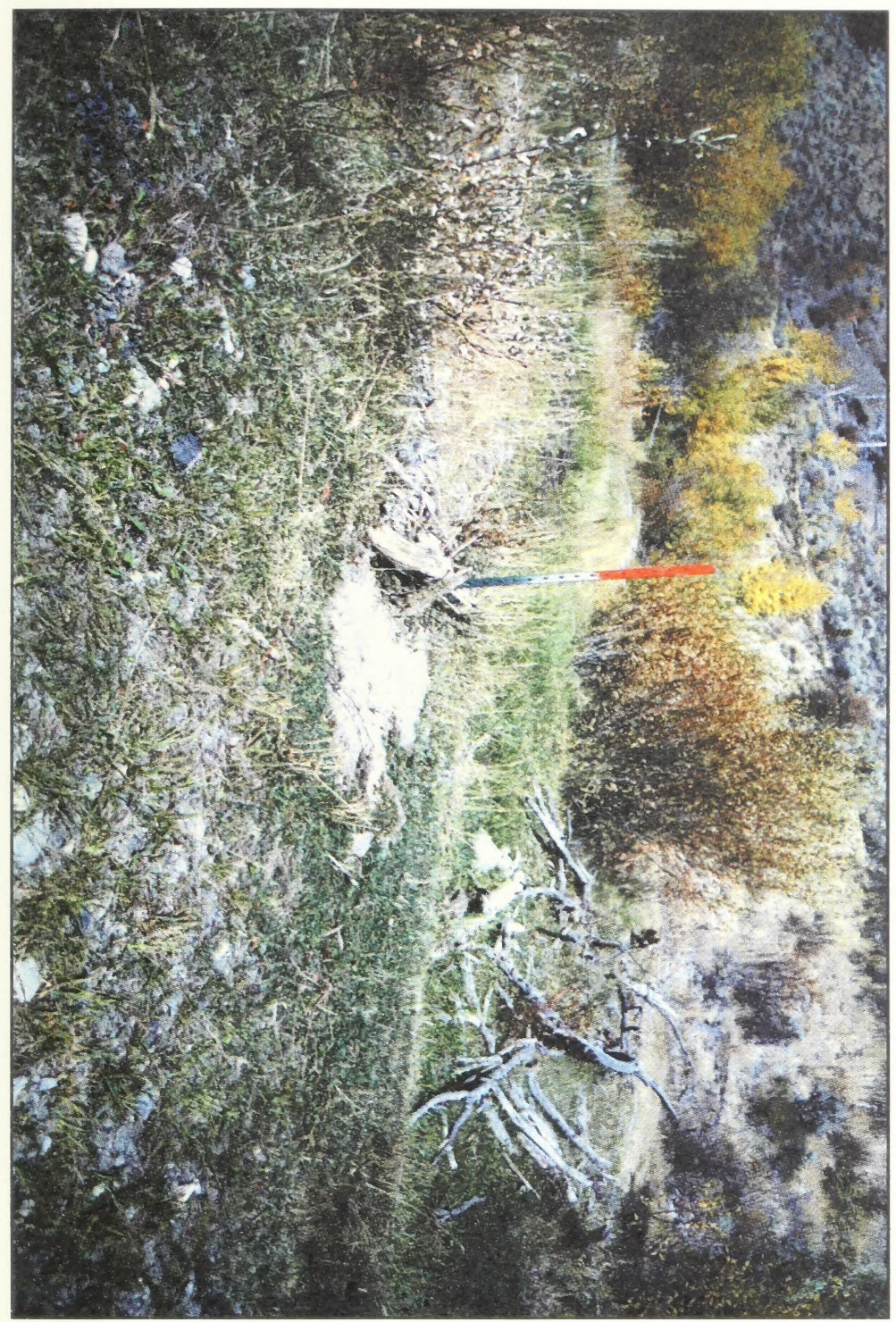

TC-KA2, Photo Point 1, Wide View, 10/3/96 


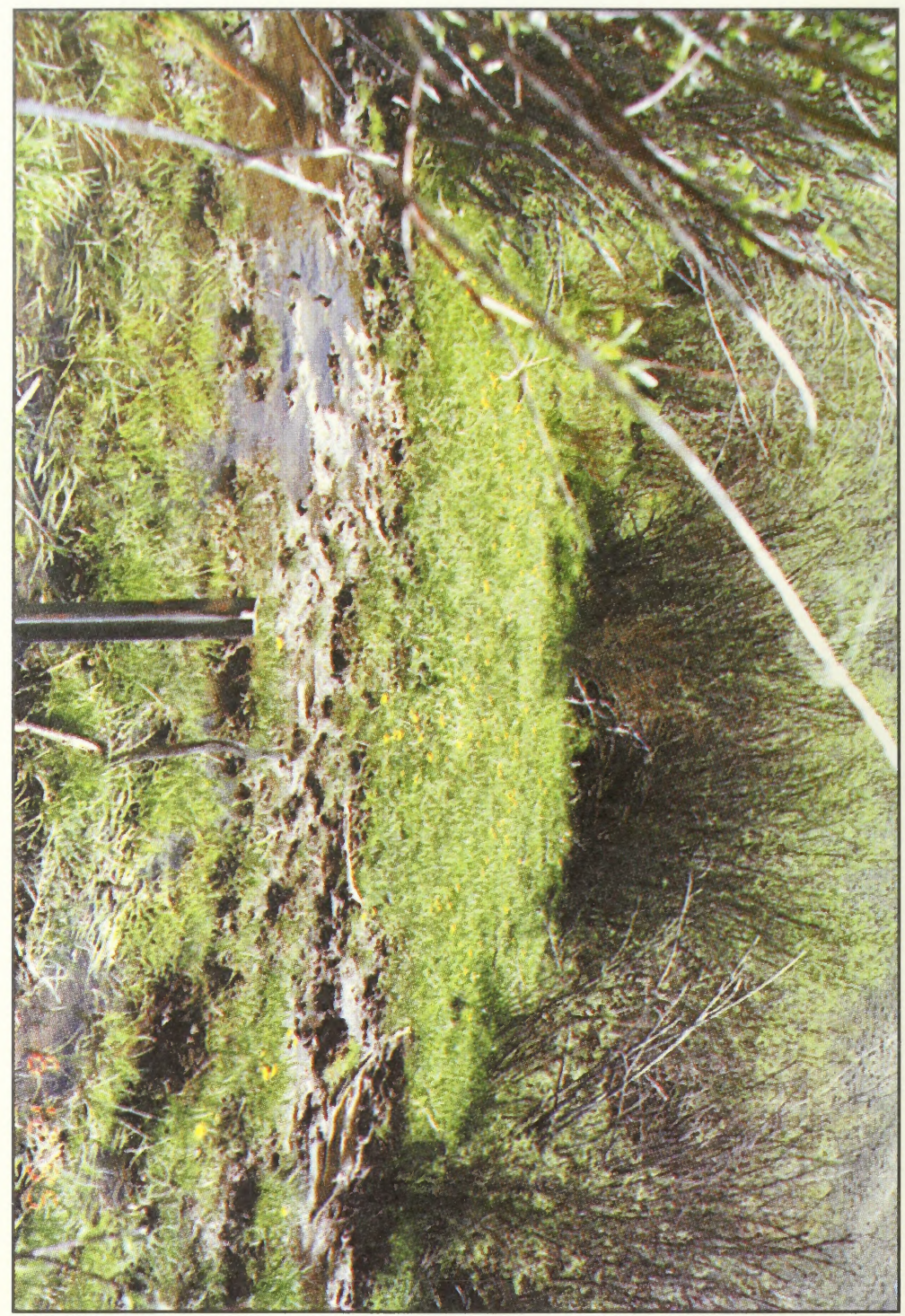

CBC-KA1, Lower Marker, Near View, 6/12/95 
Median Stubble Height - 4 inches, Sedge

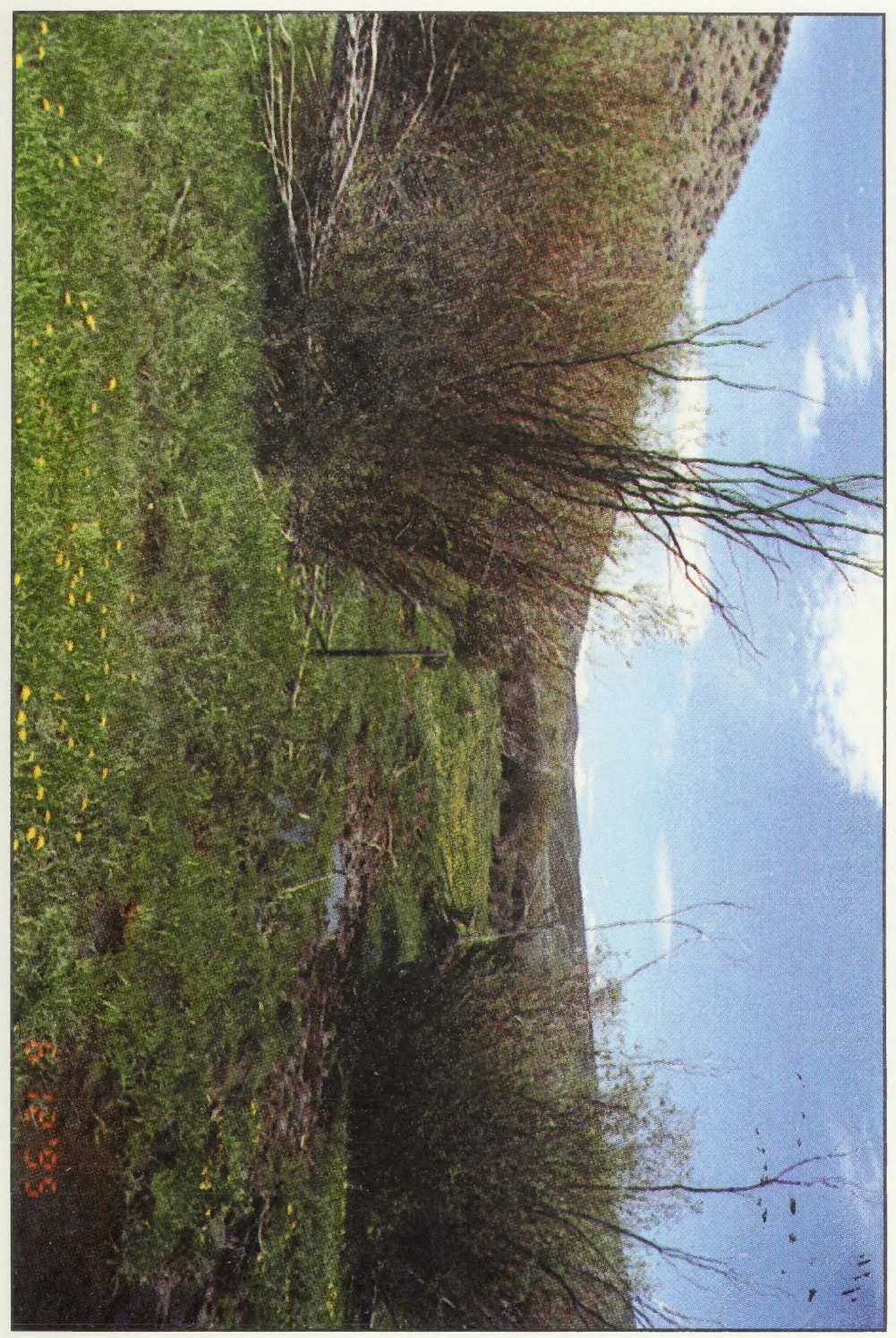

CBC-KA1, Upper Marker, Widw View,6/12/95 


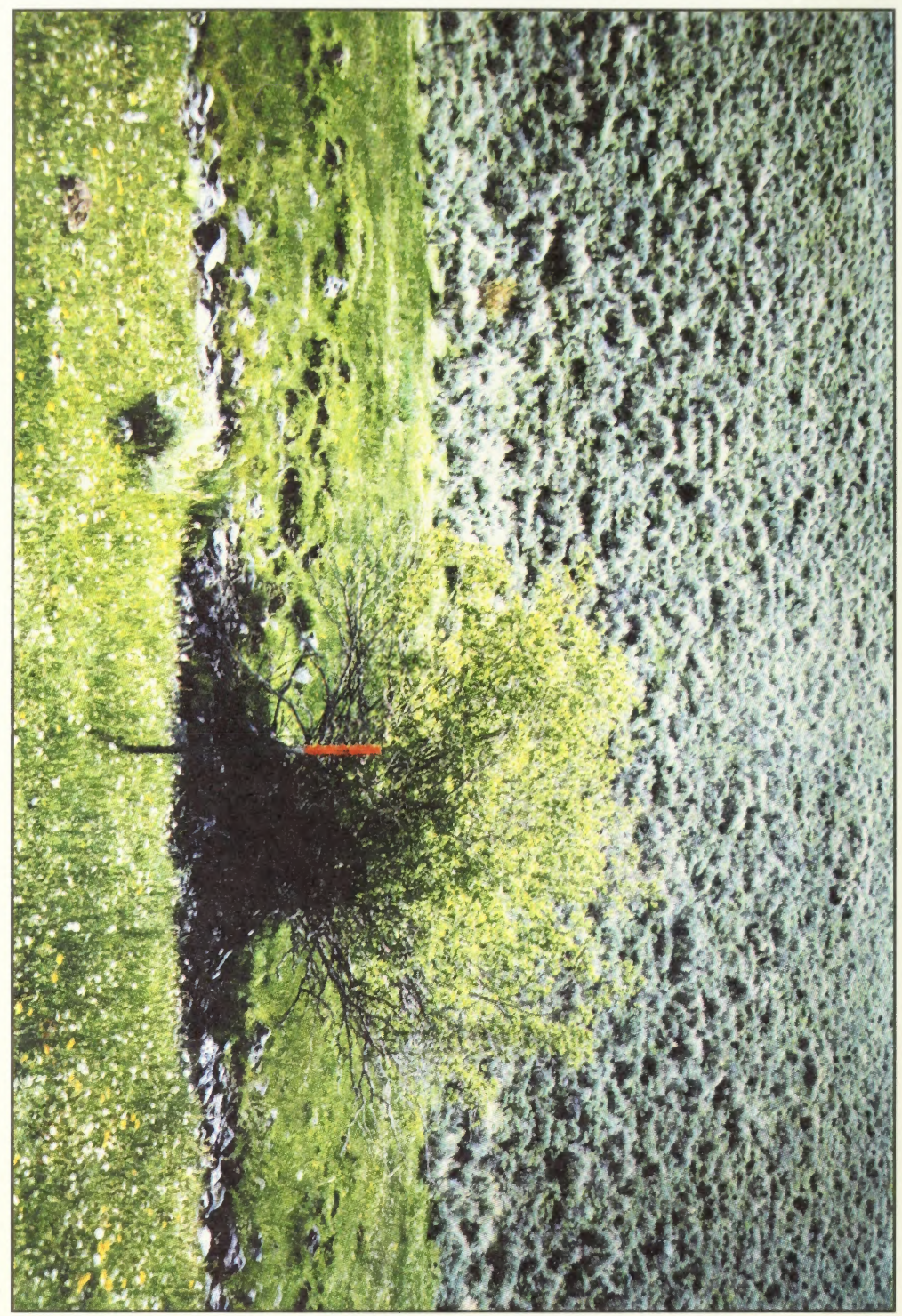

LGC-KA1, Upper Marker, Near View, 6/27/96 


\section{Median Stubble Height - 4 inches, Grass/Forb}

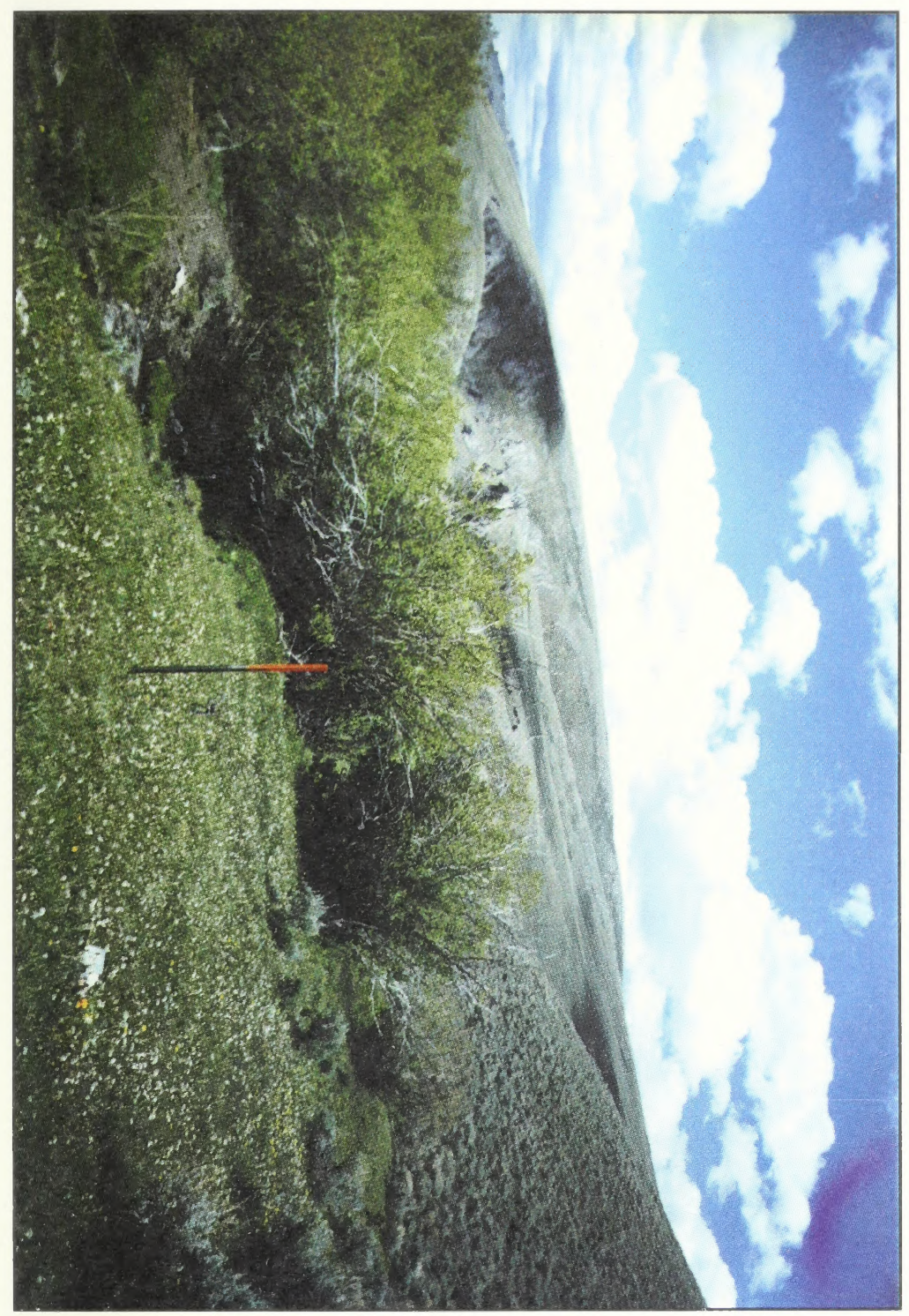

LGC-KA1, Upper Marker, Wide View, 6/27/96 


\section{Median Stubble Height - 5 inches, Sedge}



HBC-KA2, Upper Marker, Near View, 10/4/96 


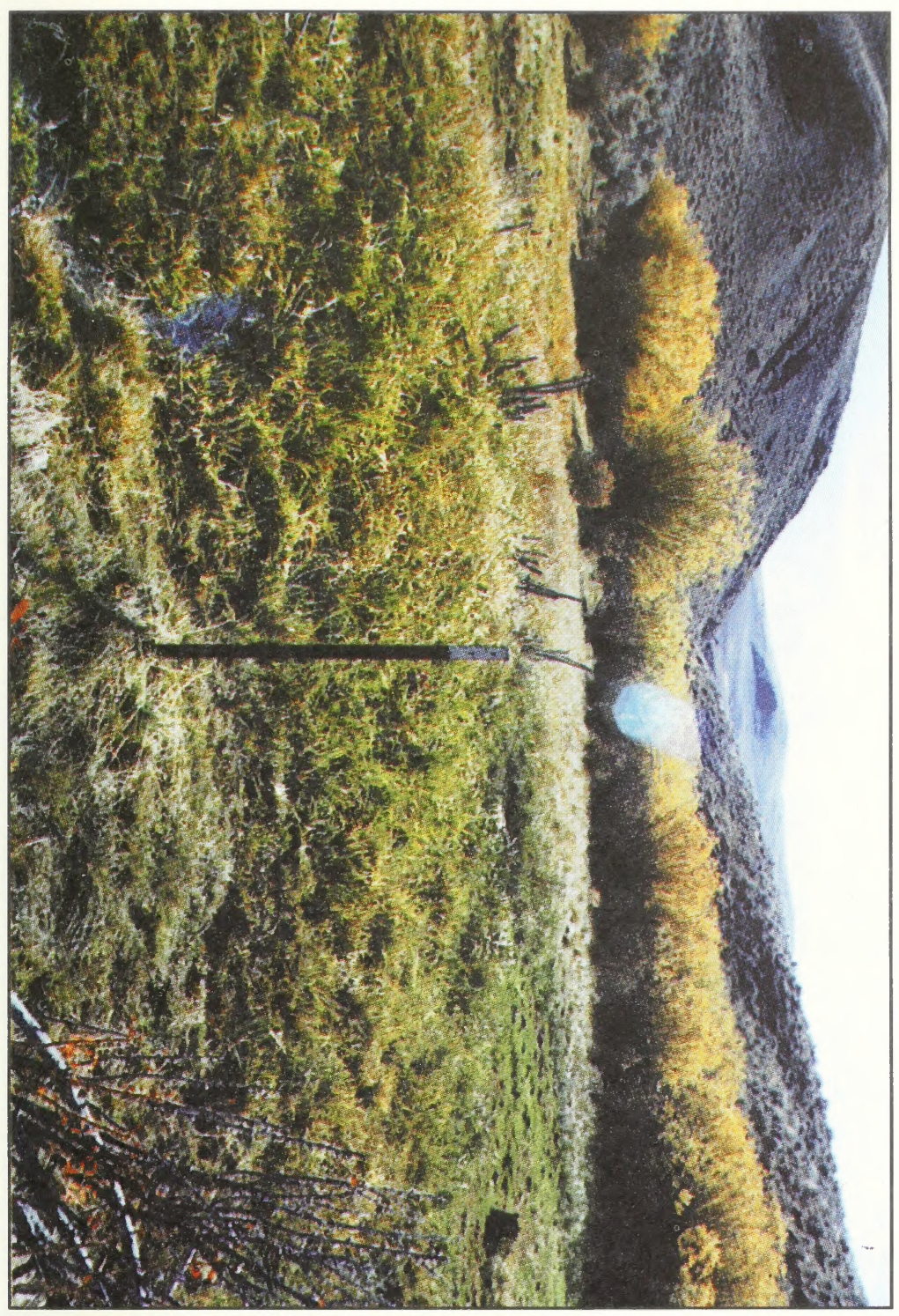

HBC-KA2, Photo Point 3, Wide View, 10/4/96 


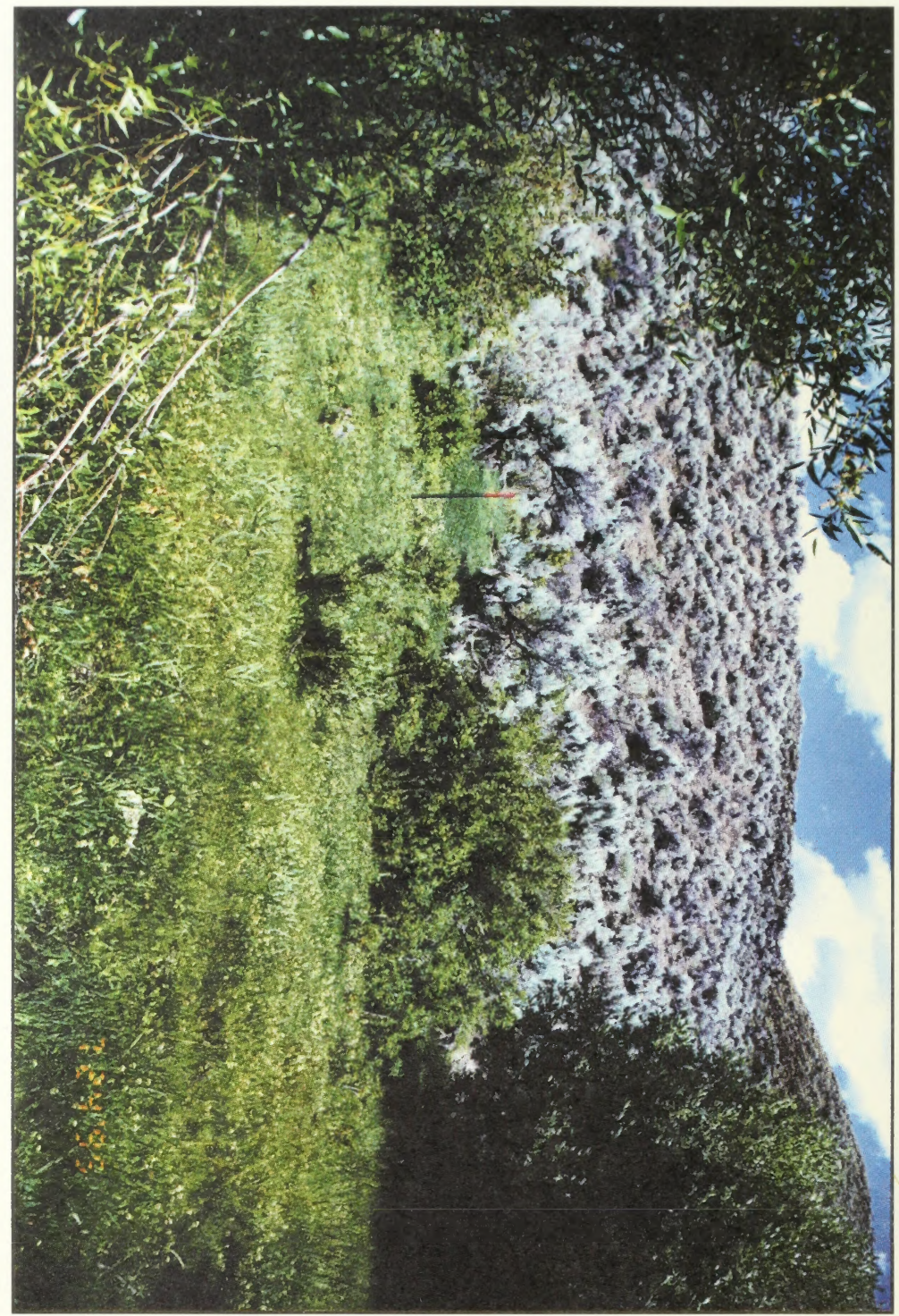

LHC-KA3, Upper Marker, Near View, 7/24/95 


\section{Median Stubble Height - 5 inches - Grass/Forb}

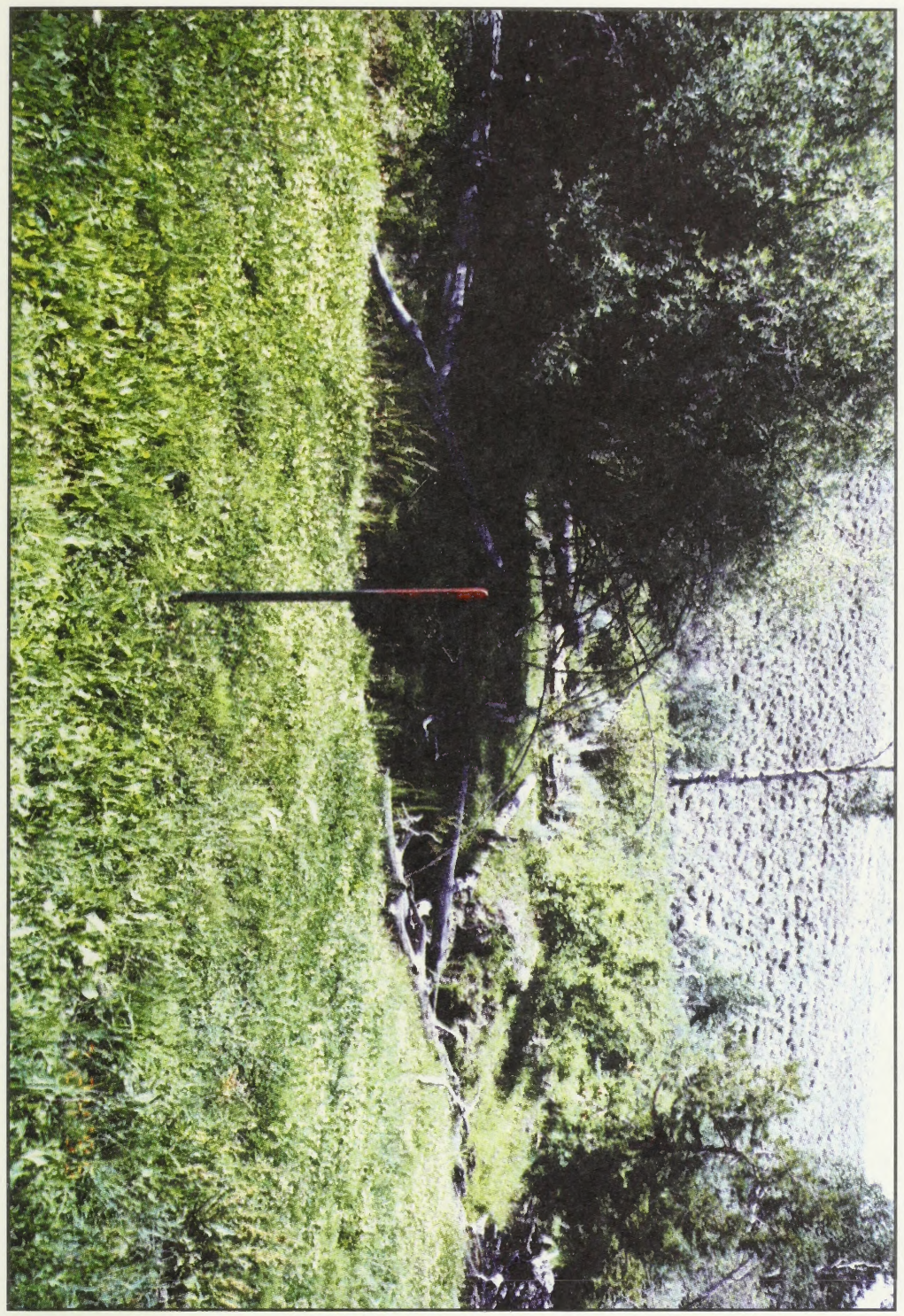

LHC-KA3, Upper Marker, Wide View, 7/24/95 


\section{Median Stubble Height - 6 inches}

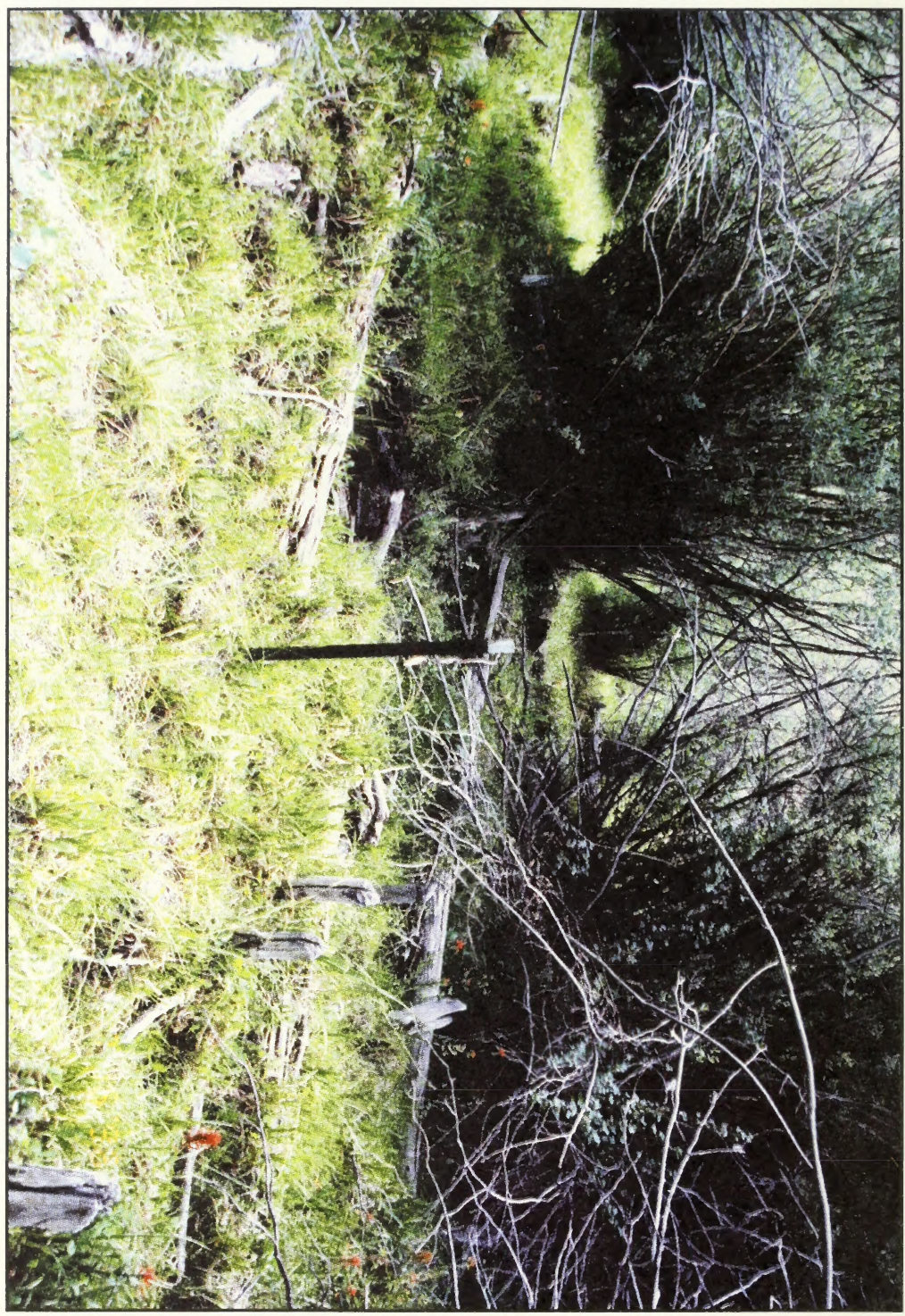

BRC-KA1, Lower Marker, Near View, 7/16/96 


\section{Median Stubble Height - 6 inches}

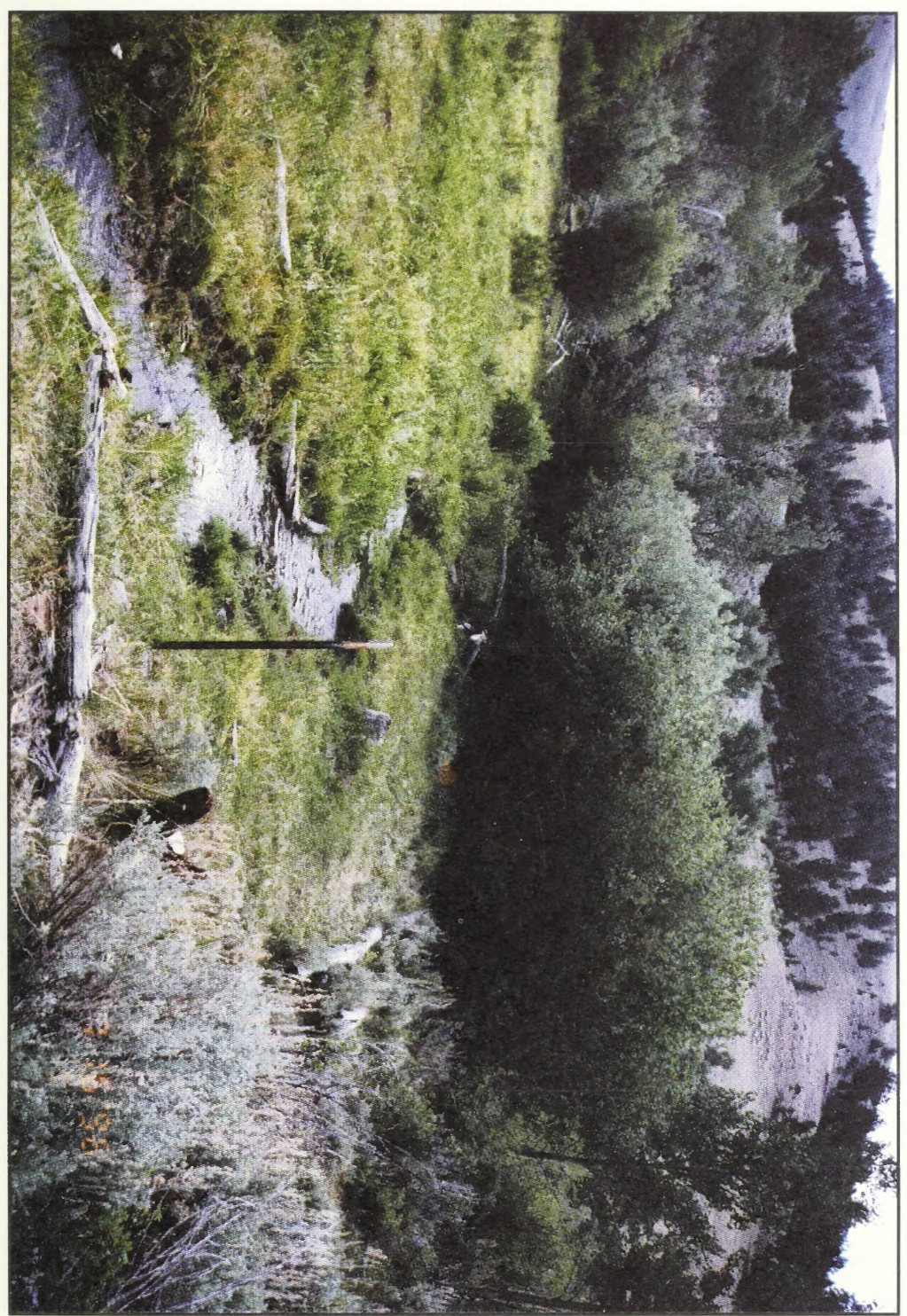

BRC-KA1, Upper Marker, Wide View, 6/13/96 


\section{Median Stubble Height - 7 inches}

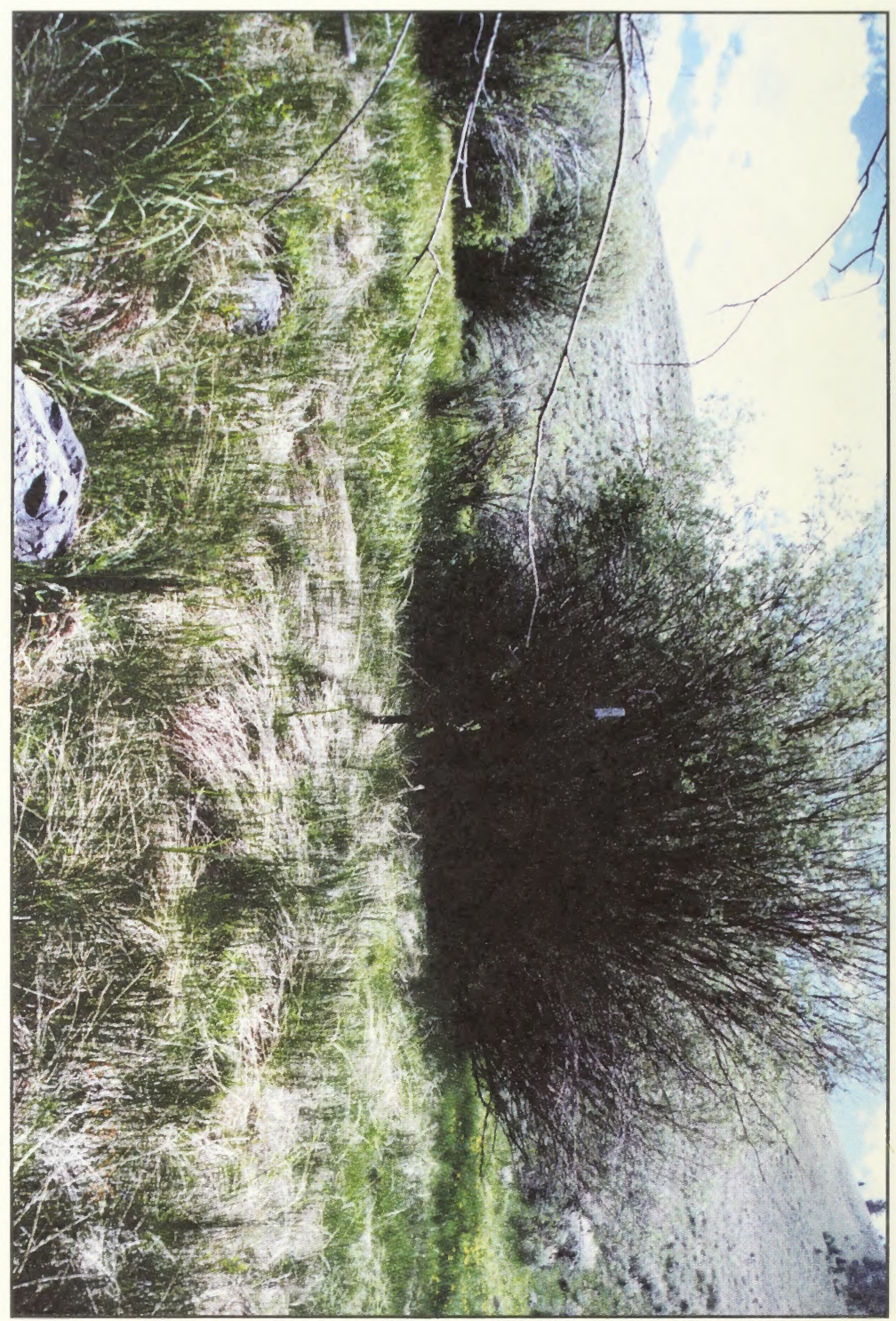

HBC-KA2, Upper Marker, Near View, 6/13/96 


\section{Median Stubble Height - 7 inches}

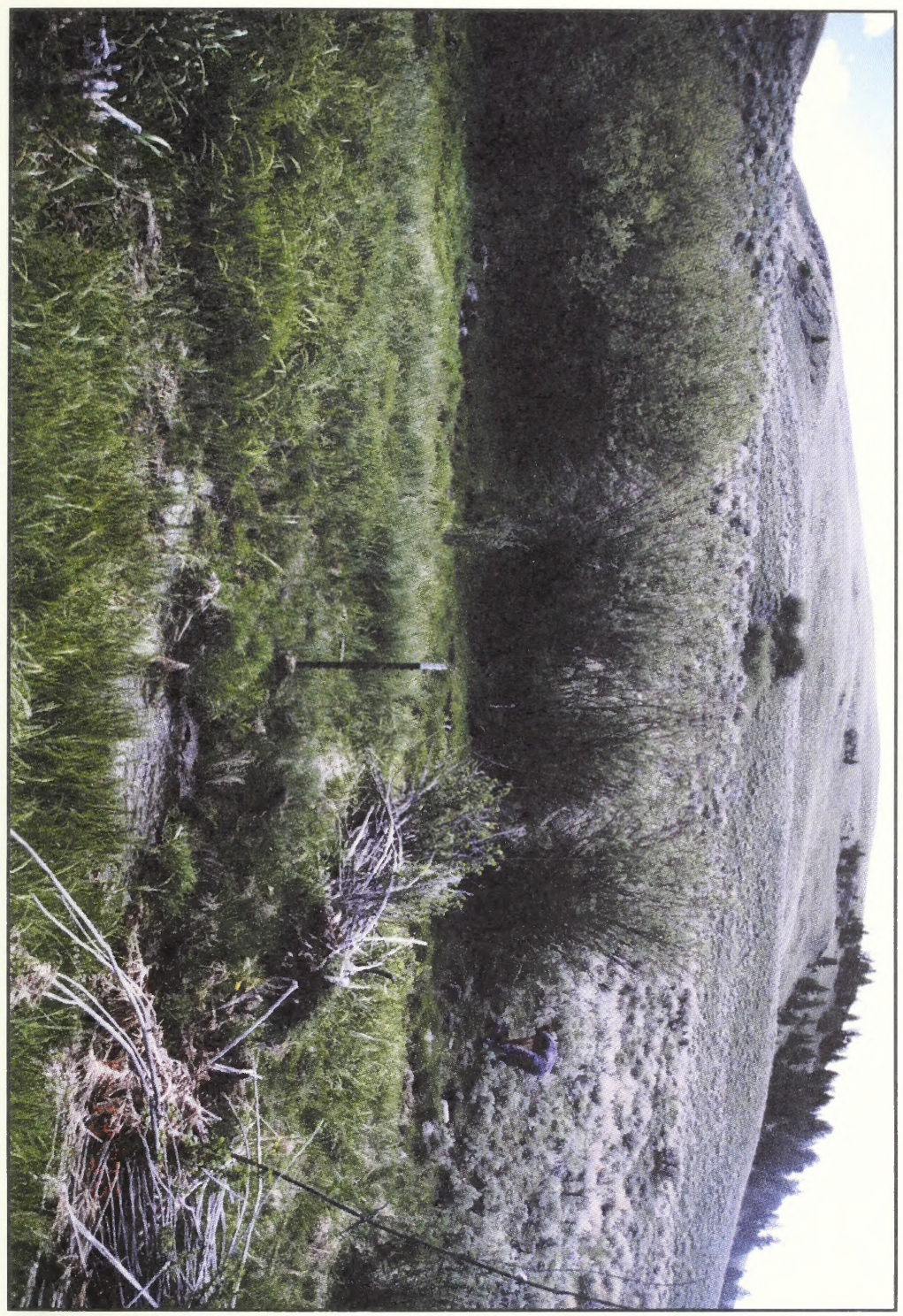

HBC-KA2, Upper Marker, Wide View, 6/13/96 


\section{Median Stubble Height - 8 inches}

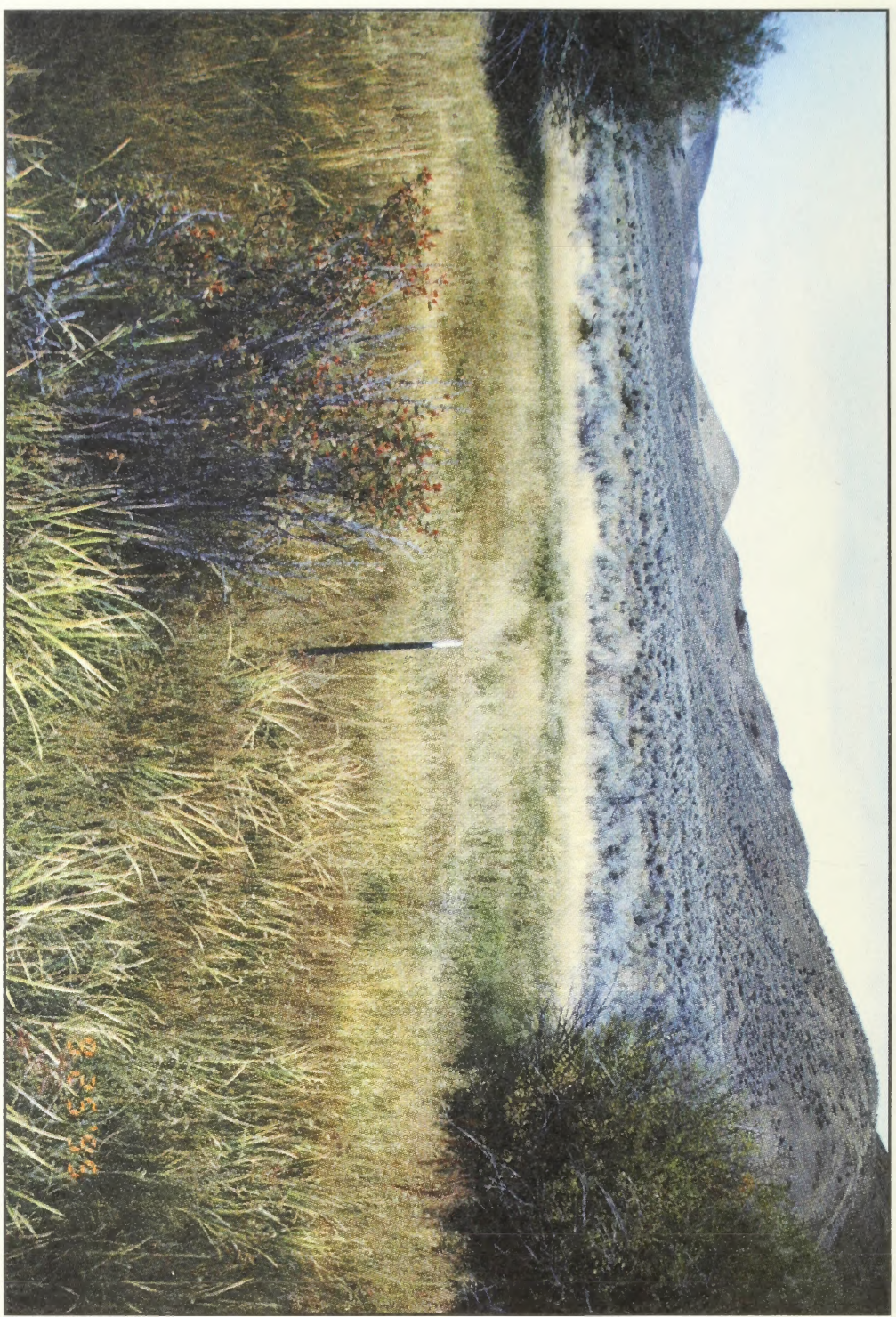

RC-KA2, Upper Marker, Near View, 9/25/95 


\section{Median Stubble Height - 8 inches}



RC-KA2 (Wide View) 9/25/95 


\section{Location Index of Key Area Sites}

$\begin{array}{lll}\text { 1.0" } & \text { CBC-KA1 } & \text { Corral Basin Creek Key Area \#1 } \\ \text { 2.0" } & \text { LHC-KA3 } & \text { Little Hat Creek Key Area \#3 } \\ \text { 3.0" } & \text { TC-KA2 } & \text { Thompson Creek Key Area \#2 } \\ \text { 4.0" Grass/Forb } & \text { LGC-KA1 } & \text { Long Creek Key Area \$1 } \\ 4.0^{\prime \prime} \text { Sedge } & \text { CBC-KA1 } & \text { Corral Basin Creek Key Area \#1 } \\ 5.0^{\prime \prime} \text { Grass/Forb } & \text { LHC-KA3 } & \text { Little Hat Creek Key Area \#3 } \\ 5.0^{\prime \prime} \text { Sedge } & \text { HBC-KA2 } & \text { Horse Basin Creek Key Area \#2 } \\ 6.0 " & \text { BRC-KA1 } & \text { Bear Creek Key Area \#1 } \\ 7.0 " & \text { HBC-KA2 } & \text { Horse Basin Creek Key Area \#2 } \\ 8.0 " & \text { RC-KA2 } & \text { Road Creek Key Area \#2 }\end{array}$

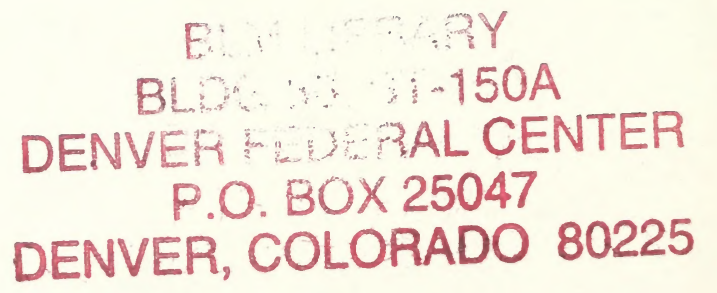




\section{BLA LIBRARY \\ BLDG 50, ST-150A \\ DENVEA FEDERAL CENTER \\ P.O. $50 \times 25047$ \\ DENVER, COLORADO 80225}

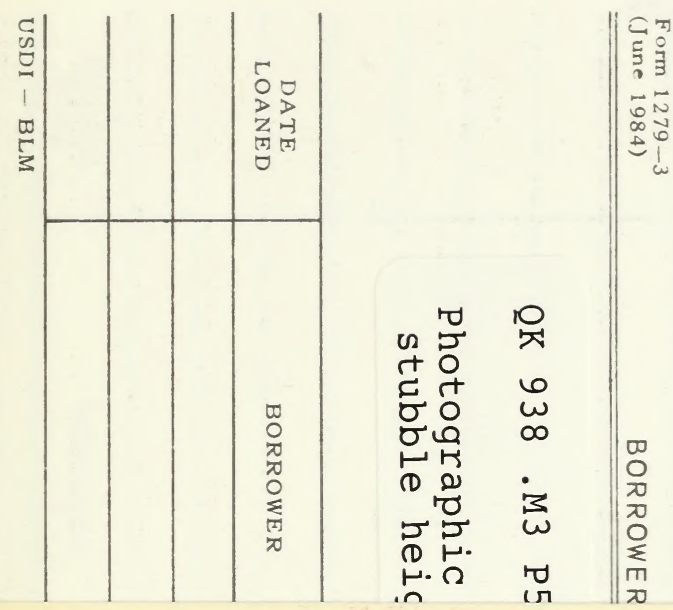

\section{BLM LIBRAPY \\ RS 150A BLDG. 50}

DENVER FEDERAL CENTER

P.O.BOX 25047

DENVER, CO 80225

QK 938 .M3 P55 1999

Photographic guide to media stubble heights 


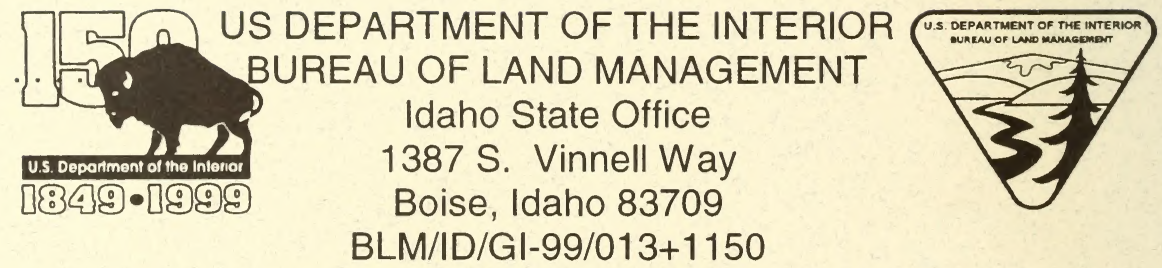

